PROCEEDINGS OF THE

AMERICAN MATHEMATICAL SOCIETY

Volume 136, Number 7, July 2008, Pages 2555-2563

S 0002-9939(08)09328-3

Article electronically published on February 21, 2008

\title{
FINITE TIME SINGULARITIES FOR A CLASS OF GENERALIZED SURFACE QUASI-GEOSTROPHIC EQUATIONS
}

\author{
HONGJIE DONG AND DONG LI \\ (Communicated by Walter Craig)
}

\begin{abstract}
We propose and study a class of generalized surface quasi-geostrophic equations. We show that in the inviscid case certain radial solutions develop gradient blow-up in finite time. In the critical dissipative case, the equations are globally well-posed with arbitrary $H^{1}$ initial data.
\end{abstract}

\section{Introduction AND MAIN RESUlts}

In this paper we consider a class of generalized surface quasi-geostrophic (SQG) equations

$$
\begin{cases}\theta_{t}+u \cdot \nabla \theta+\kappa(-\Delta)^{\gamma / 2} \theta=0 & \text { on } \mathbb{R}^{2} \times(0, \infty), \\ \theta(0, x)=\theta_{0}(x) & \text { in } \mathbb{R}^{2},\end{cases}
$$

where $\kappa \geq 0, \gamma \in(0,2]$ are fixed parameters and the velocity $u$ is related to the potential temperature $\theta$ by

$$
u=Q_{\beta}(-\Delta)^{-1 / 2} \nabla \theta
$$

with

$$
Q_{\beta}=\left(\begin{array}{cc}
\cos \beta & -\sin \beta \\
\sin \beta & \cos \beta
\end{array}\right) .
$$

Here $\beta \in[0,2 \pi)$ is also a fixed parameter. If $\beta=\pi / 2$ or $3 \pi / 2$, we recover the usual $2 \mathrm{D}$ quasi-geostrophic equation in which $u$ is divergence free. In this sense we regard (1.1) as a generalization of the usual SQG. The fractional Laplacian $(-\Delta)^{\alpha}$ for any $\alpha \in \mathbb{R}$ is defined by the Fourier transform

$$
\widehat{(-\Delta)^{\alpha}} f(\xi)=|\xi|^{2 \alpha} \hat{f}(\xi) .
$$

The case $\kappa=0$ is called inviscid SQG. When $\kappa>0$, the cases $\gamma>1, \gamma=1$ and $\gamma<1$ are called sub-critical, critical and super-critical, respectively. In this paper we will focus on the inviscid case and the critical case. Before we formulate our main results, we shall review the existing literature on the usual SQG.

Received by the editors June 11, 2007.

2000 Mathematics Subject Classification. Primary 35Q35, 82C70.

Key words and phrases. Mellin transform, finite-time singularities, quasi-geostrophic equations, global well-posedness.

The authors were partially supported by the National Science Foundation under agreement No. DMS-0111298.

(C)2008 American Mathematical Society Reverts to public domain 28 years from publication 
The inviscid SQG is derived from general quasi-geostrophic equations in the special case of constant potential vorticity and buoyancy frequency (see [13] and 23]). It is an outstanding open problem whether smooth initial data would blow up in finite time. Majda and Tabak 21] first conducted numerical simulations and found that the SQG equations with smooth initial data develop sharp fronts in the level sets of $\theta$. They conjectured the possibility of formation of a finite time singularity in $\nabla \theta$. However a more careful simulation later conducted in [7] and 22 revealed the absence of such singularity and attributed the observation in the simulation of [21] to a growth of double exponential type. Indeed Córdoba proved rigorously in [10] that the hyperbolic saddle scenario of blow up suggested in 21] is not possible. Some interesting related 1D models were also proposed and studied. Córdoba, Córdoba and Fontelos [11] [12] studied the following equations:

$$
\theta_{t}-(H \theta) \theta_{x}=-\kappa(-\Delta)^{\alpha / 2} \theta, \quad x \in \mathbb{R},
$$

where $H \theta$ is the Hilbert transform defined by

$$
H \theta:=\frac{1}{\pi} \int \frac{\theta(y)}{x-y} d y .
$$

In the inviscid case $(\kappa=0)$ they proved the existence of blow-up solutions to (1.2) for a generic class of initial data. We remark that (1.2) can be regarded as a special case of our model (1.1) if one chooses $\beta=0$ and $\theta_{0}\left(x_{1}, x_{2}\right)=\theta_{0}\left(x_{1}\right)$. Some other 1D models, all of which have some analogy with the 2D SQG and the 3D Euler equation in vorticity form, can be found in [2, 9], 6], 18, 24, 25] and [26].

The usual SQG equations with dissipation $(\kappa>0)$ have been intensively studied. The sub-critical case is well understood and the global well-posedness result is wellknown (see Constantin, Córdoba and $\mathrm{Wu}$ 8], Carrilo and Ferreira [5], Dong and Li [17, Ju [19] and the references therein). The problems of global regularity or finite-time blow-up for large initial data in the critical and supercritical case is very challenging and a breakthrough only happened recently. The global well-posedness for the critical SQG with periodic $C^{\infty}$ data was proved by Kiselev, Nazarov and Volberg in an elegant paper 20. Caffarelli and Vasseur [4 recently constructed a global regular weak solution for the critical quasi-geostrophic equations with $L^{2}$ initial data. The global well-posedness of the critical SQG for large periodic or non-periodic $H^{1}$ initial data has been recently proved by the first author and Du in 14 and [16] by adapting the method of [20] with suitable modifications. However the problem of global regularity or finite-time blow-up for large initial data in the supercritical case is still open.

We now state our main results. Our first result concerns the behavior of solutions in the inviscid case $(\kappa=0)$ with $\beta \neq \pi / 2$ or $3 \pi / 2$. It is obvious that radial solutions are steady state solutions for the usual inviscid SQG (i.e. $\beta=\pi / 2$ or $3 \pi / 2$ ). This is no longer the case for $\beta \neq \pi / 2$ or $3 \pi / 2$. In this case if we consider only the radial solutions, it is not difficult to see that (1.1) is equivalent to the following $1 \mathrm{D}$ equation by a simple scaling argument (see the beginning of Section 2 for the derivation):

$$
\theta_{t}-(T \theta) \theta_{x}=0 \quad \text { on }[0, \infty) \times(0, \infty),
$$

where

$$
(T \theta)(x):=\int_{0}^{\infty} \frac{\theta(r)}{r} g^{\prime}\left(\frac{x}{r}\right) d r
$$


and

$$
g(\xi)=\int_{0}^{2 \pi} \frac{d t}{\sqrt{\xi^{2}+1-2 \xi \cos t}} .
$$

Inspired by [1] we consider an even, positive and $C_{c}^{\infty}(\mathbb{R})$ initial profile $\theta_{0}$ such that for some constant $L>0$

$$
\max _{0 \leq x<\infty} \theta_{0}=\theta_{0}(0)=1, \quad \operatorname{Supp}\left(\theta_{0}\right) \subset[-L, L] .
$$

The local solvability of (1.3) follows from a standard argument. Since (1.3) is a transport equation, it is clear that $\theta(t, x)$ will be positive. It is also not difficult to see that the following properties hold true for $\theta(t, x)$ :

- $\operatorname{Supp}(\theta(t, \cdot)) \subset[-L, L]$.

- $\max _{x} \theta(t, \cdot)=\theta(t, 0)=1$.

- $\|\theta(t, \cdot)\|_{L^{p}} \leq\left\|\theta_{0}\right\|_{L^{p}}, 1 \leq p \leq \infty$.

Theorem 1.1 (Main theorem). Under the above assumptions for $\theta_{0}$, the solutions for (1.3) with $\kappa=0$ will always develop gradient blow-up in a finite time.

Our second result gives the global well-posedness of (1.3) when there is critical dissipation $(\gamma=1)$, which shows the effect of dissipation.

Theorem 1.2. Let $\theta_{0}$ be in either $H^{1}\left(\mathbb{R}^{2}\right)$ or $H^{1}\left(\mathbb{T}^{2}\right)$. Then there exists a unique smooth global solution to (1.1) in the case $\kappa>0, \gamma=1,0 \leq \beta<2 \pi$.

Remark 1.3. This result is perhaps a little bit surprising since in the transport term the velocity $u$ is in general not divergence-free when $\beta \neq \pi / 2$ or $3 \pi / 2$. However the techniques of 20] and [16] do not rely on the divergence-free condition and can still be applied here. A similar global well-posedness result also holds in the sub-critical dissipative case, i.e. $\gamma \in(1,2]$. In that case we require the initial data $\theta_{0}$ to be in $H^{2-\gamma}$.

The proof of Theorem 1.2 is completely similar to that of a corresponding result in [15]. Therefore we devote the rest of this paper to the proof of Theorem 1.1 .

\section{Derivation of (1.3) AND PRoOf of the MAin theOREM}

As was already mentioned, equation (1.3) is derived from (1.1) in the case where $\theta$ is radial and $\beta \neq \pi / 2,3 \pi / 2$. We now give the derivation of this fact. For radial functions $\theta$, denote $\theta(t, x)=: \tilde{\theta}(t,|x|)$. We have

$$
\begin{aligned}
(-\Delta)^{-1 / 2} \theta(t, x) & =\int_{\mathbb{R}^{2}} \frac{\tilde{\theta}(t,|y|)}{\left|(|x|, 0)-\left(y_{1}, y_{2}\right)\right|} d y \\
& =2 \pi \int_{0}^{\infty} \tilde{\theta}(r, t) g(|x| / r) d r
\end{aligned}
$$

where the function $g$ is the same as in (1.4). By this it follows almost immediately from (1.1) that

$$
u \cdot \nabla \theta=C(\beta)(T \tilde{\theta}) \tilde{\theta}_{x}
$$

where the operator $T$ is already defined in (1.3) and the constant $C(\beta) \neq 0$ if $\beta \neq \pi / 2,3 \pi / 2$. Finally we obtain (1.3) by a simple scaling argument and dropping the tildes. 
To prove our main theorem, we need the following lemma which is inspired by [11. We conjecture that it holds true for any $0 \leq \delta<1$. However due to some certain technicality (see Section 3), we are only able to prove the case $\delta=0$. But this is enough for the proof of the main theorem.

Lemma 2.1. Let $f$ be an even function on $R$ and $f \in C^{1}(R) \cap L^{\infty}(R)$ with $f(0)=0$. Then for $\delta=0$, there exists a positive constant $C_{\delta}$ such that

$$
-\int_{0}^{\infty} \frac{f^{\prime}(x)(T f)(x)}{x^{1+\delta}} d x \geq C_{\delta} \int_{0}^{\infty} \frac{1}{x^{2+\delta}} f^{2}(x) d x .
$$

Proof. By the Parseval identity for Mellin transforms, we have

$$
-\int_{0}^{\infty} \frac{f^{\prime}(x)(T f)(x)}{x^{1+\delta}} d x=-\frac{1}{2 \pi} \int_{\infty}^{\infty} \overline{A(\lambda)} B(\lambda) d \lambda=: I,
$$

where

$$
\begin{aligned}
A(\lambda) & =\int_{0}^{\infty} x^{i \lambda-\frac{1}{2}-\frac{\delta}{2}} f^{\prime}(x) d x \\
& =-\left(i \lambda-\frac{\delta+1}{2}\right) \int_{0}^{\infty} x^{i \lambda-\frac{3}{2}-\frac{\delta}{2}} f(x) d x
\end{aligned}
$$

and

$$
B(\lambda)=\int_{0}^{\infty} x^{i \lambda-\frac{3}{2}-\frac{\delta}{2}}(T f)(x) d x
$$

Now

$$
\begin{aligned}
B(\lambda) & =\int_{0}^{\infty} x^{i \lambda-\frac{3}{2}-\frac{\delta}{2}} \int_{0}^{\infty} \frac{f(r)}{r} g^{\prime}\left(\frac{x}{r}\right) d r d x \\
& =\int_{0}^{\infty} r^{i \lambda-\frac{3}{2}-\frac{\delta}{2}} f(r) d r\left(\int_{0}^{\infty} \xi^{i \lambda-\frac{3}{2}-\frac{\delta}{2}} g^{\prime}(\xi) d \xi\right) \\
& =: F(\lambda)\left(\int_{0}^{\infty} \xi^{i \lambda-\frac{3}{2}-\frac{\delta}{2}} g^{\prime}(\xi) d \xi\right),
\end{aligned}
$$

where

$$
g(\xi)=\int_{0}^{2 \pi} \frac{d t}{\sqrt{\xi^{2}+1-2 \xi \cos t}} .
$$

Therefore by the Parseval identity for Mellin transforms:

$$
\begin{aligned}
I & =\frac{1}{2 \pi} \int_{-\infty}^{\infty} M(\lambda)|F(\lambda)|^{2} d \lambda \\
& =\frac{1}{2 \pi} \int_{-\infty}^{\infty} \operatorname{Re}(M(\lambda))|F(\lambda)|^{2} d \lambda,
\end{aligned}
$$

where

$$
M(\lambda)=\left(\frac{\delta+1}{2}+i \lambda\right) \int_{0}^{\infty} \xi^{i \lambda-\frac{3+\delta}{2}} g^{\prime}(\xi) d \xi
$$

and we have used the fact that $|F(\lambda)|^{2} \operatorname{Im}(M(\lambda))$ is an odd function of $\lambda$. Now taking $\delta=0$ and using Lemma 3.3, we conclude that

$$
I \geq C \int_{-\infty}^{\infty}|F(\lambda)|^{2} d \lambda \geq C \int_{0}^{\infty} \frac{1}{x^{2+\delta}} f^{2}(x) d x,
$$


where we used the Parseval identity for Mellin transforms again:

$$
\frac{1}{2 \pi} \int_{-\infty}^{\infty}|F(\lambda)|^{2} d \lambda=\int_{0}^{\infty} \frac{1}{x^{2+\delta}} f^{2}(x) d x
$$

The lemma is proved.

Proof of the Main Theorem 1.1. By Lemma 2.1 and the properties of $\theta$, we have

$$
\begin{aligned}
\frac{d}{d t} \int_{0}^{L} \frac{1-\theta}{x} d x & =-\int_{0}^{L} \frac{(1-\theta)_{x} T(1-\theta)}{x} d x \\
& =-\int_{0}^{\infty} \frac{(1-\theta)_{x} T(1-\theta)}{x} d x \\
& \geq C_{0} \int_{0}^{\infty} \frac{(1-\theta)^{2}}{x^{2}} d x \\
& \geq C_{L}\left(\int_{0}^{L} \frac{1-\theta}{x} d x\right)^{2},
\end{aligned}
$$

where $C_{L}$ is a positive constant depending only on $L$. This implies that $J:=$ $\int_{0}^{L} \frac{1-\theta}{x} d x$ blows up at finite time. Now since

$$
J \leq \sup _{x} \frac{1-\theta}{x} \cdot L \leq L\left\|\theta_{x}\right\|_{L^{\infty}}
$$

we conclude that $\left\|\theta_{x}\right\|_{L^{\infty}}$ also blows up in finite time, and our theorem is proved.

\section{The LOWER Bound of $\operatorname{Re}(M(\lambda))$}

We show in this section that $\operatorname{Re}(M(\lambda))$ is bounded below by a positive absolute constant. Recall that

$$
M(\lambda)=\left(\frac{\delta+1}{2}+i \lambda\right) \int_{0}^{\infty} \xi^{i \lambda-\frac{3+\delta}{2}} g^{\prime}(\xi) d \xi
$$

Let $\alpha=\frac{\delta+1}{2}$. Integrating by parts, we get

$$
M(\lambda)=\left(\lambda^{2}+\alpha^{2}+\alpha+\lambda i\right) \int_{0}^{\infty} \xi^{i \lambda-\alpha-2}(g(\xi)-g(0)) d \xi .
$$

For $\xi \neq 1$, we expand

$$
\begin{aligned}
& g(\xi)-g(0) \\
& =\int_{0}^{2 \pi}\left(\frac{\frac{1}{\sqrt{\xi^{2}+1}}}{\sqrt{1-\frac{2 \xi}{\xi^{2}+1} \cos t}}-1\right) d t \\
& =\frac{1}{\sqrt{\xi^{2}+1}} \sum_{k \geq 0} \frac{(2 k-1) ! !}{(2 k) ! !}\left(\frac{2 \xi}{\xi^{2}+1}\right)^{k} \int_{0}^{2 \pi} \cos ^{k} t d t-2 \pi \\
& =2 \pi\left(\sum_{k \geq 1} \frac{(4 k-1) ! !}{(4 k) ! !} \frac{(2 k-1) ! !}{(2 k) ! !}(2 \xi)^{2 k}\left(\xi^{2}+1\right)^{-2 k-\frac{1}{2}}+\frac{1}{\sqrt{\xi^{2}+1}}-1\right) .
\end{aligned}
$$


Substituting this series into (3.1), using Fubini's theorem and integrating by parts in the last term, we have

$$
\begin{aligned}
M(\lambda)= & \left(\lambda^{2}+\alpha^{2}+\alpha+\lambda i\right) \pi \sum_{k \geq 0} \frac{(4 k-1) ! !}{(4 k) ! !} \frac{(2 k-1) ! !}{(2 k) ! !} \frac{4^{k}}{\Gamma\left(2 k+\frac{1}{2}\right)} \\
& \cdot \Gamma\left(k+\frac{\alpha-i \lambda+2}{2}\right) \Gamma\left(k-\frac{\alpha-i \lambda+1}{2}\right),
\end{aligned}
$$

where for $k=0$ we define $(-1) ! !=1$, and $\Gamma(\cdot)$ is the Gamma function. It is most convenient to choose $\alpha=1 / 2$ since in this case we have

$$
\begin{aligned}
& \Gamma\left(k+\frac{\alpha-i \lambda+2}{2}\right) \Gamma\left(k-\frac{\alpha-i \lambda+1}{2}\right) \\
& =\left(k+\frac{1}{4}-\frac{i \lambda}{2}\right)\left(k-\frac{3}{4}-\frac{i \lambda}{2}\right)\left|\Gamma\left(k-\frac{3}{4}+\frac{i \lambda}{2}\right)\right|^{2} .
\end{aligned}
$$

We assume henceforth that $\alpha=\frac{1}{2}$ and write

$$
\begin{aligned}
& \operatorname{Re}(M(2 \lambda)) \\
& =C \sum_{k \geq 0} a_{k}(\lambda)\left(\left(16 \lambda^{2}+3\right)\left(\left(k+\frac{1}{4}\right)\left(k-\frac{3}{4}\right)-\lambda^{2}\right)+2 \lambda^{2}\left(2 k-\frac{1}{2}\right)\right),
\end{aligned}
$$

where $C$ is a positive absolute constant and

$$
\begin{aligned}
a_{k}(\lambda) & =\frac{(4 k-1) ! !}{(4 k) ! !} \frac{(2 k-1) ! !}{(2 k) ! !} \frac{4^{k}}{\Gamma\left(2 k+\frac{1}{2}\right)}\left|\Gamma\left(k-\frac{3}{4}+\frac{i \lambda}{2}\right)\right|^{2} \\
& =\frac{1}{(k !)^{2} \Gamma\left(\frac{1}{2}\right)}\left|\Gamma\left(k-\frac{3}{4}+\frac{i \lambda}{2}\right)\right|^{2}>0 .
\end{aligned}
$$

Denote

$$
F(k, \lambda)=a_{k}(\lambda)\left(\left(16 \lambda^{2}+3\right)\left(\left(k+\frac{1}{4}\right)\left(k-\frac{3}{4}\right)-\lambda^{2}\right)+2 \lambda^{2}\left(2 k-\frac{1}{2}\right)\right) .
$$

Lemma 3.1. Assume $\alpha=\frac{1}{2}$. There exists a positive constant $C_{1}$ such that for any $\lambda \geq 1$ we have

$$
\sum_{k \geq 100 \lambda^{2}} F(k, \lambda) \geq C_{1} \lambda
$$

Proof. We use the letter $C$ to denote absolute constants which may vary from line to line. First by using Stirling's formula we have

$$
\sum_{k \geq 100 \lambda^{2}} F(k, \lambda) \geq C \sum_{k \geq 100 \lambda^{2}} \frac{\lambda^{2}}{k^{3 / 2}} \frac{\left|\Gamma\left(k-\frac{3}{4}-i \lambda\right)\right|^{2}}{\left|\Gamma\left(k-\frac{3}{4}\right)\right|^{2}} .
$$

By using the representation of $\Gamma(z)$,

$$
\Gamma(z)=\lim _{n \rightarrow \infty} \frac{n ! n^{z}}{z(z+1) \cdots(z+n)},
$$

it is easy to show that for any $k \geq 100 \lambda^{2}$,

$$
\frac{\left|\Gamma\left(k-\frac{3}{4}-i \lambda\right)\right|^{2}}{\left|\Gamma\left(k-\frac{3}{4}\right)\right|^{2}} \geq C
$$

where the constant $C$ does not depend on $\lambda$. The lemma is proved by noting that $\sum_{k \geq 100 \lambda^{2}} k^{-3 / 2} \geq C \lambda^{-1}$. 
Observe that $F(k, \lambda) \geq 0$ whenever $k \geq \lambda+1$. We have the following lemma.

Lemma 3.2. Let $\lambda \geq 5$ and $N=[\lambda]$. Then for any integer $1 \leq k \leq N+2$, we have

$$
F(N+k+1, \lambda)+F(N-k+2, \lambda) \geq 0 .
$$

Proof. We begin by observing the following easy inequality which holds for any $1 \leq k \leq N+2, \lambda \geq 5$ :

$$
\begin{aligned}
& \left(16 \lambda^{2}+3\right)\left(\lambda^{2}-\left(N-k+\frac{9}{4}\right)\left(N-k+\frac{5}{4}\right)\right)+2 \lambda^{2}\left(\frac{1}{2}-2(N-k+2)\right) \\
& \leq\left(16 \lambda^{2}+3\right)\left(\left(N+k+\frac{5}{4}\right)\left(N+k+\frac{1}{4}\right)-\lambda^{2}\right)+2 \lambda^{2}\left(2(N+k+1)-\frac{1}{2}\right) .
\end{aligned}
$$

In fact it is enough to prove the case $k=1$ and we leave it as an exercise to interested readers. Now it suffices for us to show

$$
a_{N-k+2}(\lambda) \leq a_{N+k+1}(\lambda)
$$

or

$$
\frac{\left(N+\frac{1}{4}\right)^{2}+N^{2}}{(N+2)^{2}} \prod_{j=1}^{k-1} \frac{\left(N+\frac{1}{4}-j\right)^{2}+N^{2}}{(N+2-j)^{2}} \cdot \frac{\left(N+\frac{1}{4}+j\right)^{2}+N^{2}}{(N+2+j)^{2}} \geq 1 .
$$

It can be simply checked that for $N \geq 5$ and any $j \geq 0$

$$
\frac{\left(N+\frac{1}{4}\right)^{2}+N^{2}}{(N+2)^{2}} \geq 1, \quad \frac{\left(N+\frac{1}{4}-j\right)^{2}+N^{2}}{(N+2-j)^{2}} \cdot \frac{\left(N+\frac{1}{4}+j\right)^{2}+N^{2}}{(N+2+j)^{2}} \geq 1 .
$$

The lemma is proved.

We are now ready to prove our main lemma concerning the lower bound on $\operatorname{Re}(M(\lambda))$.

Lemma 3.3. Assume $\alpha=\frac{1}{2}$. Then there exists a constant $C_{1}>0$ such that

$$
R e(M(\lambda)) \geq C_{1}(1+|\lambda|), \quad \forall \lambda \in R .
$$

Proof. By (3.2), observe that $\operatorname{Re}(M(\lambda))=\operatorname{Re}(M(|\lambda|))$. Assume first $\lambda \geq 5$. Then by Lemma 3.1 and Lemma 3.2, we get

$$
\operatorname{Re}(M(2 \lambda)) \geq \sum_{k \leq 2 N+3} F(k, \lambda)+\sum_{k \geq 100 \lambda^{2}} F(k, \lambda) \geq C \lambda .
$$

Next we consider the remaining case $0 \leq \lambda<5$. Clearly

$$
\operatorname{Re}(M(2 \lambda)) \geq \sum_{k \leq 7} F(k, \lambda)+\min _{\lambda \in[0,5]} F(100, \lambda)
$$

It suffices for us to show that

$$
\sum_{k \leq 7} F(k, \lambda) \geq 0
$$


A straightforward computation gives

$$
\begin{aligned}
& \sum_{k \leq 7} F(k, \lambda)=\left|\Gamma\left(-\frac{3}{4}-i \lambda\right)\right|^{2} \frac{1}{109099041266073600} \\
& \cdot\left(13532187772216575+60858017636694624 \lambda^{2}\right. \\
& +232195801218409728 \lambda^{4}+1714841444714835968 \lambda^{6} \\
& +734756426151297024 \lambda^{8}+95075778545319936 \lambda^{10} \\
& +4031405671579648 \lambda^{12}-18819204513792 \lambda^{14} \\
& \left.-4509715660800 \lambda^{16}-68719476736 \lambda^{18}\right) .
\end{aligned}
$$

We note that

$$
\begin{aligned}
& 4031405671579648 \lambda^{12} \geq 4509715660800 \lambda^{16}+68719476736 \lambda^{18}, \\
& 5075778545319936 \lambda^{10} \geq 18819204513792 \lambda^{14},
\end{aligned}
$$

for any $\lambda \in[0,5)$. Our lemma is proved.

\section{ACKNOWLEDGMENTS}

We thank two anonymous referees for helpful comments and informing us of the recent paper by Balodis and Córdoba [1] in which a similar result is obtained by different methods.

\section{REFERENCES}

[1] Balodis, P., Córdoba, A., Inequality for Riesz transforms implying blow-up for some nonlinear and nonlocal transport equations, Adv. Math. 214 (2007) no. 1, 1-39.

[2] Bertozzi, A.L., Majda, A.J., Vorticity and Incompressible Flow, Cambridge Univ. Press, Cambridge, UK (2002). MR 1867882(2003a:76002)

[3] Beale, J.T., Kato, T., and Majda, A., Remarks on the breakdown of smooth solutions for the 3-D Euler equations, Comm. Math. Phys. 94 (1984), no. 1, 61-66. MR763762 (85j:35154)

[4] Caffarelli, L., Vasseur, A., Drift diffusion equations with fractional diffusion and the quasigeostrophic equation, preprint.

[5] Carrilo, J.A., Ferreira, L.C.F., Asymptotic behavior for the sub-critical dissipative quasigeostrophic equations, preprint.

[6] Chae, D., Córdoba, A., Córdoba, D., and Fontelos, M.A., Finite time singularities in a 1D model of the quasi-geostrophic equation, Adv. Math. 194 (2005), 203-223. MR 2141858 (2006a:76116)

[7] Constantin, P., Nie, Q., and Schörghofer, N., Nonsingular surface quasi-geostrophic flow, Phys. Lett. A 241 (1998), 168-172. MR1613907 (99a:76031)

[8] Constantin, P., Córdoba, D., and Wu, J., On the critical dissipative quasi-geostrophic equation, Indiana Univ. Math. J. 50, (2001), 97-107. MR1855665(2002h:35246)

[9] Constantin, P., Lax, P., and Majda, A., A simple one-dimensional model for the threedimensional vorticity equation, Comm. Pure Appl. Math. 38 (1985), 715-724. MR812343 (87a:76037)

[10] Córdoba, D., Nonexistence of simple hyperbolic blow-up for the quasi-geostrophic equation, Ann. of Math. 148 (1998), 1135-1152. MR.1670077 (2000j:76020)

[11] Córdoba, A., Córdoba, D., and Fontelos, M.A., Formation of singularities for a transport equation with nonlocal velocity, Ann. of Math. 162 (2005) (3), 1377-1389. MR2179734 (2007b:35011)

[12] Córdoba, A., Córdoba, D., and Fontelos, M.A., Integral inequalities for the Hilbert transform applied to a nonlocal transport equation, J. Math. Pures Appl. 86 (2006) (6), 529-540. MR2281451 (2007k:35040) 
[13] Constantin, P., Majda, A.J., and Tabak, E., Formation of strong fronts in the 2-D quasigeostrophic thermal active scalar. Nonlinearity 7 (1994), no. 6, 1495-1533. MR1304437 (95i:76107)

[14] Dong, H., Higher regularity for the critical and super-critical dissipative quasi-geostrophic equations, arXiv:math.AP/0701826.

[15] Dong, H., On the well-posedness for a transport equation with nonlocal velocity, preprint.

[16] Dong, H., Du, D., Global well-posedness and a decay estimate for the critical dissipative quasi-geostrophic equation in the whole space, to appear in Discrete Contin. Dyn. Syst., arXiv:math.AP/0701828.

[17] Dong, H., Li, D., Spatial analyticity of the solutions to the sub-critical dissipative quasigeostrophic equations, submitted.

[18] De Gregorio, S., A partial differential equation arising in a 1D model for the 3D vorticity equation, Math. Methods Appl. Sci. 19 (1996), 1233-1255. MR.1410208 (97i:76031)

[19] Ju, N., Dissipative quasi-geostrophic equation: local well-posedness, global regularity and similarity solutions, Indiana Univ. Math. J. (2006), in press.

[20] Kiselev, A., Nazarov, F., and Volberg, A., Global well-posedness for the critical 2D dissipative quasi-geostrophic equation, Invent. Math. 167 (2007), no. 3, 445-453. MR2276260

[21] Majda, A.J., Tabak, E.G., A two-dimensional model for quasi-geostrophic flow: comparison with the two-dimensional Euler flow, Physica D. 98 (1996), 515-522. MR.1422288 (97g:86005)

[22] Ohkitani, K., Yamada, M., Inviscid and inviscid-limit behavior of a surface quasi-geostrophic flow, Phys. Fluids 9 (1997), 876-882. MR1437554 (97m:76032)

[23] Pedlosky, J., Geophysical Fluid Dynamics, Springer-Verlag, New York, 1987.

[24] Sakajo, T., On global solutions for the Constantin-Lax-Majda equation with a generalized viscosity term, Nonlinearity 16 (2003), 1319-1328. MR.1986297 (2004d:76029)

[25] Schochet, S., Explicit solutions of the viscous model vorticity equation, Comm. Pure Appl. Math. 41 (1986), 531-537. MR840339 (87h:35322)

[26] Yang, Y., Behavior of solutions of model equations for incompressible fluid flow, J. Differential Equations 125 (1996), 133-153. MR.1376063(97b:76045)

Division of Applied Mathematics, Brown University, 182 George Street, Providence, RHODE ISLAND 02912

E-mail address: hdong@brown.edu

School of Mathematics, Institute for Advanced Study, Einstein Drive, Princeton, NEW JERSEY 08540

E-mail address: dongli@math.ias.edu 